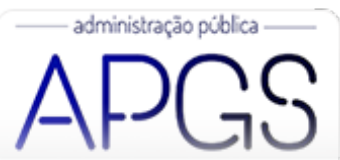

Administração Pública e Gestão Social ISSN: 2175-5787

apgs@ufv.br

Universidade Federal de Viçosa

Brasil

\title{
Enfoque de derecho y políticas de juventud. Un análisis del Programa de Respaldo a Estudiantes de Argentina
}

\author{
Analia Elizabeth, Otero \\ Enfoque de derecho y políticas de juventud. Un análisis del Programa de Respaldo a Estudiantes de Argentina \\ Administração Pública e Gestão Social, vol. 12, núm. 3, 2020 \\ Universidade Federal de Viçosa, Brasil \\ Disponible en: http://www.redalyc.org/articulo.oa?id=351563312007
}

Esta obra está bajo una Licencia Creative Commons Atribución-NoComercial-SinDerivar 3.0 Internacional. 


\title{
Enfoque de derecho y políticas de juventud. Un análisis del Programa de Respaldo a Estudiantes de Argentina
}

\author{
Foco da lei e políticas da juventude. Uma análise do Programa de Apoio ao Estudante da Argentina \\ Focus of law and youth policies. An analysis of the Argentina Student Support Program \\ Otero Analia Elizabeth \\ Redalyc: http://www.redalyc.org/articulo.oa? \\ IICSAL (FLACSO-CONICET), Argentina \\ $\mathrm{id}=351563312007$ \\ aotero14@gmail.com \\ Recepción: 17 Julio 2018 \\ Aprobación: 29 Noviembre 2019 \\ Publicación: 01 Julio 2020
}

\section{RESUMEN:}

Entre las políticas públicas dirigidas a apoyar la inclusión de los jóvenes en Argentina durante el año de 2014 hemos presenciado el lanzamiento de un programa de transferencia condicionada (PTC), él Programa de Respaldo a Estudiantes de Argentina PRO.G.RES.AR (en adelante Progresar). Este ha surgido en un marco donde se impulsó el enfoque de derechos y comenzó a cobrar relevancia la orientación hacia un paradigma de protección social integral. El objetivo del artículo es analizar el referido programa a partir de retomar como base los hallazgos de una investigación donde se trabajó a partir de una estrategia metodológica cualitativa con entrevistas semi-estructuradas, recogiendo la opinión de diversos actores (gestores y expertos académicos) y documental, retomando decretos y normativas del programa. Los hallazgos arrojan dos elementos centrales: La dinámica propuesta muestra avances con relación a la extensión de las intervenciones destinadas a la franja etaria de 18 a 24 años y persisten limitaciones en materia de políticas de juventud.

Palabras ClaVe: Juventud, Política pública, Inclusión Social.

\section{Resumo:}

Entre as políticas públicas destinadas a apoiar a inclusão de jovens no mercado de trabalho na Argentina em 2014, assistimos ao lançamento de um programa de transferência condicional (PTC), o Programa de Apoio ao Estudante da Argentina PRO.G.RES.AR, que surgiu em uma estrutura na qual a abordagem de direitos foi promovida e a orientação para um paradigma abrangente de proteção social começou a ganhar relevância. $\mathrm{O}$ objetivo do artigo é analisar o programa mencionado, com base na retomada dos resultados de uma prévia investigação com base em uma estratégia metodológica qualitativa, com entrevistas semiestruturadas, reunindo a opinião de vários atores (gerentes e especialistas acadêmicos) e documentando os decretos e regulamentos do programa. Os resultados possuem dois elementos centrais: A dinâmica proposta mostra progresso em relação à extensão das intervenções voltadas para a faixa etária de 18 a 24 anos eas limitações nas políticas de juventude persistem.

PalaVras-chaVe: Juventude, Políticas Públicas, Inclusão Social.

\begin{abstract}
:
Among the public policies designed to support the inclusion of young people in the labour market in Argentina during 2014, we have witnessed the launch of a conditional transfer program (PTC), the Student Support Program of Argentina PRO.G.RES.AR (en forward Progress), which emerged in a framework where the rights approach was promoted and the orientation towards a paradigm of integral social protection began to gain relevance. The objective of the article is to analyze a previously launched program based on retaking the findings of an investigation based on a qualitative methodological strategy with semi-structured interviews gathering the opinion of various actors (managers and academic experts) and documentary taking up decrees and program regulations. The findings have two central elements: The proposed dynamics show progress in relation to the extension of the interventions aimed at the age group from 18 to 24 years old, and limitations on youth policies persist.
\end{abstract}

KEYwORDs: Youth, Public Policy, Social Inclusion. 


\section{INTRODUCCIÓN}

Los jóvenes, aún más aquellos que cuentan con menores recursos, resultan uno de los sectores poblacionales más afectados por fenómenos como el avance de la precarización, informalidad, exiguas posibilidades de empleo y, escaso acompañamiento para la finalización de trayectorias educativo-laborales exitosas. (Bertranou \& Casanova, 2015). No obstante, durante el último periodo las políticas destinadas a las juventudes parecen estar ganando cierto protagonismo en Argentina, según el Informe de la Comisión Económica para América Latina y el Caribe y el Organismo Internacional de Juventud para Iberoamérica (2014), la inversión social dirigida al sector alcanzó el 3.3\% (porcentaje de Producto Bruto Interno), lo cual lo posiciona como aquel país con la mayor inversión en políticas públicas de juventud en Sudamérica. Bajo este encuadre ambos organismos destacan el Programa de Respaldo a Estudiantes de Argentina (PRO.G.RES.AR) como ejemplo de:

[...] una de las formas de inversión a través de la cual el Estado se vale para apoyar a jóvenes de menos recursos (económicos) en el marco de la heterogeneidad de las trayectorias juveniles, de tal manera que logren concretar sus proyectos de vida." (CEPAL/OIJ, 2014, p. 30).

Este texto presenta un análisis del Progresar, como el proyecto fue nombrado más tarde, creado por Decreto (PE) No 84/2014 (BO 27/01/2014) realizado en el marco del proyecto Políticas públicas y jóvenes Apuestas, balances e interrogantes de cara al futuro (2015-2017), que tuvo como objetivo contribuir al campo de los estudios de juventud a través de una investigación sobre las intervenciones públicas destinadas a atender sus problemáticas en el contexto actual. Giro en torno a describir, caracterizar y analizar el referido programa como una estrategia destinada a promover la inclusión educativa-laboral. Atendió a profundizar en las innovaciones, límites y obstáculos de la medida considerando su ubicación en el esquema de intervenciones públicas destinadas a dicho sector poblacional. Y, postulo analizar su contenido técnico, así como indagar en las opiniones de actores (gestores/académicos expertos) sobre el mismo, partiendo del supuesto que tal sondeo permite identificar pautas claves en la trama de abordaje de lo joven y sus vacíos pendientes.

La estrategia metodológica adoptada ha sido cualitativa y documental, utilizando fuentes primarias y secundarias de información. Como fuentes secundarias se analizó el decreto de creación, en base al cual se describieron los rasgos técnico-normativos centrales de la medida. Con el objeto de indagar en la opinión de actores se diseñó una muestra no representativa e intencional de gestores y expertos académicos aplicándose entrevistas semi-estructuradas durante el 2016 y, orientados por una estrategia analítica reflexiva se detectaron nudos neurálgicos y tensiones de interés trazando tanto posiciones transversales como puntos controversiales entre las opiniones.

En lo que va del texto, en un primer apartado y en diálogo con el contexto regional, bosquejaremos las principales transformaciones en materia de las políticas públicas, en particular, de educación-trabajo destinadas a los jóvenes en Argentina. Luego apuntaremos rasgos centrales de la investigación y la estrategia metodológica con la que se ha trabajado. Posteriormente, presentaremos el Progresar dando cuenta de sus características centrales en base al decreto de origen y, sin pretensiones de ser excluyente repasaremos autores que desde diferentes perspectivas lo han abordado. Avanzaremos después, en el análisis en base a los testimonios recabados. Finalmente, concluiremos con una breve síntesis sobre el recorrido realizado.

\section{UNA POLÍTICA EN CONTEXTO}

En Argentina, más que un sistema integrado, existe un conjunto de diversas y fragmentadas estrategias de intervención estatal destinadas al sector joven y es históricamente endeble la institucionalidad que detenta el campo. Como se ha observado: 
Hace más de tres décadas los expertos en la temática sostienen que uno de los ejes críticos en este campo tiene que ver con la falta de integralidad de las acciones estatales para responder de forma articulada y eficaz al conjunto de las problemáticas vivenciadas por la juventud. En este sentido, el predominio de políticas sectoriales, con escasa y (o) inadecuada coordinación, parece estar dando respuestas inacabadas a las problemáticas sociales, laborales, educativas, familiares y de salud, etc. (Otero, 2017, p. 63).

También, históricamente, se ha dado prioridad a las estrategias dirigidas a los jóvenes de los sectores más vulnerables que mostraron hasta aquí exiguos avances. En el grueso de los casos las políticas públicas, tuvieron como objetivo explícito la inclusión social y, en términos generales, han enfocado en el binomio educación-trabajo/capacitación-empleo. Experiencias del tipo han sido, entre muchos otros el Proyecto Joven (1993/01) ${ }^{[1]}$ y el Programa Incluir (2004/07). Puede decirse que no son recientes los debates sobre dispositivos enfocados en educación-trabajo, sin embargo, casi a finales de la década de los años 2000 las intervenciones estatales se han reforzado a través del lanzamiento de nuevos programas. Lo cual se ha dado en el marco de transformaciones de envergadura en las concepciones y en el esquema de políticas públicas donde el enfoque de derecho tomó centralidad y a diferencia de periodos antecesores se verificó un impulso notable por ampliar el tradicional esquema de seguridad social y extender la cobertura de programas destinados a niños y jóvenes ${ }^{[2]}$.

Como telón de fondo de este planteo, distintos autores han considerado que desde principios del siglo XXI el esquema de política pública en América Latina, presenta cierto viraje orientado por la prioridad que adquiere el enfoque de derechos e inclusión social. Una consecuencia central de la nueva orientación es que, desplaza la concepción de los destinatarios como beneficiarios de lo público por una basada en los derechos ciudadanos donde el estado tiene la responsabilidad fundamental como garante de estos. En el encuadre del derecho, surgirán transformaciones trascendentes, por ejemplo, intervenciones públicas "más integrales", dado que no solo son destinadas a los individuos, sino que la familia pasa a ser el núcleo central de buena parte de las medidas. (Feijoó \& Poggi, 2014).

Esto se aunará a las tendencias surgidas desde mediados de los noventa cuando cobrará lugar el modelo de transferencias condicionadas PTC un tipo de política pública cuya implementación comprende la entrega directa de efectivo entendida como un soporte a grupos que sufren determinadas carencias, siendo un apoyo para la atención de gastos básicos. Estas transferencias tendrán una contraparte particular dado que implican que los sujetos tendrán que cumplir con ciertas exigencias en materia de acceso a bienes públicos como controles de salud, asistencia educativa, por ejemplo. En el campo de la política pública, existe una amplia bibliografía a cerca de los programas de Transferencia de Ingresos que se han extendido en la región de América Latina a partir de mediados de la década de los años 1990. México y Brasil han sido precursores en el lanzamiento de programas de este tipo. (Feijoó \& Poggi, 2014).

Fundamentalmente, el signo característico del mencionado viraje en el presente siglo ha sido el reposicionamiento del estado orientado a asumir la responsabilidad ante las garantías sociales básicas, entre otros, a través de PTC. Bajo este esquema, el concepto de protección social deviene la base sobre la cual pivotearon nuevas políticas basadas en el enfoque de derechos promotoras de formatos y articulaciones sectoriales que marchan en línea con la idea de dar respuestas integrales ante el carácter indivisible de los derechos. (Jacinto, 2016).

Por su parte, el abordaje conceptual de protección social integral ha cobrado cierta prioridad en oposición a las políticas contra la pobreza de los noventa centradas en la focalización. (Cechini \& Martínez, 2011; Cechini, 2014; Kaplan \& Levy, 2014; Ocampo \& Gómez-Arteaga, 2017).

En correspondencia al esquema regional, en Argentina, en lo que respecta a políticas de niñez y juventud, en los años 2000 nos hallamos ante un cambio que impulsa este nuevo paradigma con entramados de diseños técnico-institucionales que hacen hincapié en derechos. Ante ello, algunos autores sugieren que estamos ante un panorama ampliado de la protección social cuyas manifestaciones se verifican, entre otras, en la extensión de medidas de cobertura. Por ejemplo, en la población de niños cobra expresión en la Asignación Universal 
por Hijo (AUH) de 2009, Decreto PEN No 1602/09 [3] más la Asignación Universal por Embarazo (AUE). La AUH, es básicamente una asignación destinada a las niñas (o niños) y adolescentes cuyos padres, tutores o curadores se encuentren desocupados o se desempeñen en la economía informal por un salario menor al Mínimo Vital y Móvil. Lo sustantivo de esta medida ha sido que posibilitó ampliar el régimen de asignaciones familiares, incluyendo trabajadores informales y desempleados, modificando así el esquema tradicional de cobertura de la seguridad social orientada al trabajador formal. Es decir, logro incluirse como parte del subsistema no contributivo dentro del régimen de asignaciones familiares, consolidándose como una cobertura del ciclo obligatorio de educación formal hasta los 18 años ${ }^{[4]}$.

En el caso del sector poblacional joven, esto se expresó en la reconfiguración de las políticas públicas orientadas al sector que sostienen un tono más integral y en la magnitud que adquirieron programas como: el Programa Jóvenes con Más y Mejor Trabajo (PJMYMT) (2008) [5] y el Progresar (2014) ${ }^{[6]}$, ambos destinados a jóvenes de 18 a 24 años.

En rigor, durante los años 2000 la centralidad de las acciones enfocadas en educación y trabajo siguieron dominando el escenario, no obstante, la amplitud de la cobertura, el acento en la finalización de los niveles de estudios y la incorporación de distintas herramientas de acompañamiento a las trayectorias, comenzaron a ser aspectos claves en las discusiones y acciones estatales. Siguiendo a Jacinto, desde el 2010 asistimos a una reformulación de las orientaciones en las políticas de educación, formación y empleo donde cobra prioridad la terminalidad educativa, la orientación sociolaboral y la capacitación, si bien la empleabilidad aparece como una constante en los programas se observan variaciones en este nuevo milenio.

En el marco de un enfoque de protección social integral, la misma concepción sobre la empleabilidad adquiere un carácter multidimensional que comprende aspectos de orientación sociolaboral, apoyo para la finalización de niveles formales de estudio, pasantías, capacitaciones, etc. Es decir, distintas acciones y servicios se integran como parte de lo entendido bajo el paraguas de la empleabilidad ampliando su espectro comprendiendo e incluyendo desde competencias mínimas para un empleo hasta saberes para la ciudadanía, lo cual básicamente responde a un quiebre que tiene que ver con el reconocimiento de los requerimientos mínimos del mercado de trabajo respecto a los títulos del nivel medio como condición de inserción laboral, al tiempo que refuerza el reconocimiento de la complejidad e intensidad de los diversos fenómenos en tensión que atraviesan los sectores poblacionales jóvenes de cara al mercado de trabajo actual.

Las manifestaciones de tales reformulaciones se expresaron en parte en la ampliación de las medidas, la extensión de la cobertura, como así también, en que las intervenciones adquirieran una reconfiguración de la mano del diseño de políticas activas que se contraponen a las netamente asistencialistas que dominaron el periodo anterior. Ejemplo de ello presenciamos el lanzamiento de el PJMYMT y el Progresar.

Sin embargo, el signo más potente y enfático respecto a las intervenciones estuvo puesto en la escolarización a través de la promoción de nuevos formatos escolares y el impulso de los Centros Educativos de Nivel Secundario (CENS) que son las instituciones de Secundaria de Adultos mayores de 18 años destinadas a personas que quieran retomar o iniciar los estudios secundarios y cuentan con un plan de estudios de tres años de duración. A ello se sumó la implementación de un nuevo plan nacional para finalización de los estudios primarios y secundarios el Plan FINES en $2008^{[7]}$. Resta decir que en este viraje de las políticas quedó implícito el creciente esfuerzo por ampliar las limitaciones del formato escolar típico, en pos de alcanzar mejores logros educativos. Tal fortalecimiento marcho en línea con una política institucional donde se establecieron nuevas normativas en educación. Por un lado, durante el 2005, asistimos a la sanción de la Ley de Formación Técnico Profesional No 26058/05 y posteriormente a la Ley de Educación Nacional No 26206 del 2006, que extendió el piso de educación obligatoria a los 12 años, abarcando así el nivel secundario.

En breve palabras, en el marco de las reconfiguraciones reseñadas se da una intensificación de las medidas dirigidas a los jóvenes impulsando y fortaleciendo su construcción como sujetos de derecho, en esta trama se implementa el Progresar que veremos más de cerca en los próximos apartados luego de describir aspectos centrales de la investigación. 


\section{SOBRE LA INVESTIGACIÓN Y LA ESTRATEGIA METODOLÓGICA}

El interés académico en los estudios a cerca de los y las jóvenes contemporáneos marchan en paralelo a las transformaciones de distinta índole social, productiva, económica, cultural, etc., parte de nuestra realidad cotidiana. Un epicentro del debate gira en torno a las complejidades que revisten las vinculaciones al mercado de trabajo que constituyen un límite a la hora de concretar su independencia y autonomía. Actualmente la terminalidad educativa, el combate al desempleo y la precariedad laboral cobraron lugar destacado. Los esfuerzos de las políticas públicas muestran dispares y exiguos avances, así como tensiones fundamentales para pensar acciones de alcance ante las problemáticas que están afectando aguda y desigualmente las trayectorias de las nuevas generaciones de allí el intento por ampliar las discusiones en materia de política pública.

Como indicáramos, este texto se realizó en el marco del proyecto Políticas públicas y jóvenes - Apuestas, balances e interrogantes de cara al futuro (2015-2017). En particular, en este artículo se analizó el programa Progresar desde su creación hasta el año 2018, abordando su contenido técnico e indagando en las opiniones de actores gestores y expertos académicos, entendiendo que ello puede permitir ampliar la comprensión sobre las orientaciones dadas e identificar pautas claves en la trama de las políticas públicas abonando materiales para la reflexión respecto al esquema de protecciones sociales existentes.

El supuesto es que, dada la complejidad de la relación entre la configuración de las trayectorias de los jóvenes y las posibilidades de acción efectiva de las políticas públicas, su lectura requiere un abordaje que permita articular la trama de dimensiones estructurales, así como discutir las intervenciones implementadas recogiendo no solo las normativas sino también analizando la opinión de actores que intervienen y (o) analizan las medidas referidas al sector. Por ello, como parte del plan se indagó en la opinión de actores intervinientes en la elaboración del diseño o gestión y (o) puesta en práctica del programa PROG.R.ES.AR. Entendiendo, además, que estas proveerán un material de interés en la vinculación de este programa y su articulación en el escenario político-económico, así como en la agenda de la política pública. Más ampliamente, la investigación buscó abordar una cuestión, que si bien no es enteramente novedosa es susceptible a las variaciones del contexto socio histórico. Lo cual, en este caso significa que la orientación del análisis cobra connotaciones específicas - en buena medida novedosas -, a la luz de las estrategias de programas lanzados en la última década.

Tal como anunciáramos en la introducción, para este análisis se utilizaron complementariamente fuentes primarias y secundarias de información. Por un lado, con el objeto de indagar en la opinión de actores clave, se aplicaron entrevistas semiestructuradas a una muestra no representativa e intencional, a gestores y expertos académicos del campo de la juventud. La labor de campo implicó un primer encuadramiento de la organización del trabajo, el diseño y reajuste de la herramienta de relevamiento, la ubicación de los actores y la efectiva realización en campo. La guía de pautas de entrevista comprendió una batería de preguntas abiertas, incluyendo dos bloques temáticos centrales de interés: Uno enfocó en distintos aspectos de las políticas de juventud en el contexto actual de las políticas públicas del país. Otro se orientó a recoger opiniones acerca del programa bajo análisis. Se priorizó en los lineamientos, orientación, actividades programáticas, componentes, diseño e implementación, así como en particularidades de la propuesta. En el respecto a las políticas públicas de juventud se consideró la importancia de las problemáticas a dar respuesta y su prioridad, los vacíos existentes, las encrucijadas de debate, etc. De este modo el trabajo de campo y las orientaciones respecto a las entrevistas se articularon bajo dos ejes centrales vinculados a las políticas públicas de juventud y al programa referido. Como hilo conductor se buscó trazar posiciones en el respecto al esquema de protecciones sociales, el programa y su implementación. Para la construcción de la muestra de gestores y expertos académicos se realizó un primer relevamiento de gestores del programa a través de búsquedas en los organismos oficiales origen del programa y páginas oficiales de referencia, de igual modo se relevó información de actores académicos especializados en la elaboración de textos sobre el programa. 
Tras el primer contacto con los actores se convocó a la realización de una entrevista personal. Debe aclararse que dado la etapa de recambios efectuados en la presidencia de la Nación Argentina con la asunción de Mauricio Macri a fines del 2015 y en los organigramas de organismos públicos en 2016, se redujo la posibilidad de localizar y entrevistar algunos actores intervinientes en las etapas iniciales del programa. No obstante, la muestra final se compuso de 13 registros, se entrevistaron a 8 mujeres y 5 varones, de los cuales 6 trabajaban (o trabajaron) involucrados en la gestión del programa referido, y 7 investigadores académicos que habían elaborado textos y (o) informes del programa o de las políticas públicas referidas al sector. Cabe aclarar aquí que se mantuvo el anonimato de los entrevistados a través de la utilización de nombres ficticios, por la misma razón no se indicó la institución laboral de pertenecía. El proceso de análisis se guio por una estrategia analítica reflexiva profundizando en puntos comunes y divergencias sobre el Progresar y la orientación de las políticas públicas destinadas al sector.

Por otro lado, como fuentes secundarias, se analizaron documentos oficiales, se ha retomado el Decreto (PE) No 84/2014 (BO 27/01/2014). Básicamente, se consideraron aspectos claves entre otros, normativa; orientación; prescripción; acciones programáticas, etc., prestando particular atención a los fundamentos de la propuesta; objetivos generales y específicos; población destinataria; metodologías; articulación con sectores sociales y organismos públicos; rasgos innovadores, puntos de conexión y (o) divergencia con otras políticas. Se procuro un análisis del contenido técnico y la caracterización del programa como estrategia de apoyo a las trayectorias educativas y laborales. También se retomaron brevemente aspectos centrales del Decreto (PE) No 90/2018 que introduce transformaciones sustantivas: Lo transforma en un programa de becas con origen en el Ministerio de Educación.

\section{La trama del Progresar}

El Progresar originalmente se orientó a proporcionar inclusión educativa y componentes de políticas activas de empleo, sumando a la prestación de seguridad económica. Su objetivo ha sido generar oportunidades de inclusión y desde su mismo enmarque técnico-normativo parte de concebir a los jóvenes como sujetos de derechos encuadrándose, en particular, en el acceso al derecho a la educación.

Más específicamente como versa en el decreto de creación el programa procura generar nuevas oportunidades de inclusión social para jóvenes en situación de vulnerabilidad ante el reconocimiento ineludible de:

\footnotetext{
Que la problemática juvenil tiene múltiples facetas que deben ser contempladas a la hora de abordar la temática, evitando caer en categorizaciones simplificadas y estigmatizantes, entre las que pueden mencionarse la necesidad de un acceso real y flexible a la oferta educativa, la provisión de estrategias públicas de cuidado para los jóvenes que tienen niños a cargo y el acompañamiento en una inserción laboral de calidad. Que por ello resulta conveniente la creación del programa con el objeto de finalizar la escolaridad obligatoria, iniciar o facilitar la continuidad de una educación superior y realizar experiencias de formación y/o practicas calificantes en ambientes de trabajo. Decreto (PE) No 84/2014 (BO 27/01/2014).
}

De modo que partiendo del decreto origen, el programa comulga con generar oportunidades de inclusión social y laboral a través de acciones integradas atendiendo a la problemática juvenil en múltiples facetas, pero básicamente en función de promover acceso real y flexible a la oferta educativa y acompañamiento para una inserción laboral de calidad. Por lo cual, en rigor siguiendo la línea de políticas antecesoras continúa enfocando en el binomio educación-trabajo.

Básicamente, se trata de una transferencia monetaria directa con condicionalidades y desde su normativa prescribe una vinculación sectorial e interinstitucional, en términos de articulación entre organismos de origen del programa con distintos ministerios y organismos institucionales, al tiempo que formula explícitamente una articulación con otros programas nacionales en implementación (salud[9]-empleo). Entre otros, estos rasgos promueven ciertas distinciones en el respecto a programas similares previos. Lo cual lo hizo destacable como una política con signos de viraje respecto a las vigentes del tipo. 
En relación con los destinatarios, pueden acceder todos los jóvenes argentinos de entre 18 y 24 años inclusive, que acrediten estar inscriptos o asistan a instituciones educativas habilitadas que, al momento de la solicitud no trabajan, trabajan informalmente o tienen un salario menor al mínimo vital y móvil y su grupo familiar posee iguales condiciones.

Es un programa de alcance nacional, en el cual los jóvenes pueden inscribirse en todos los niveles educativos habilitados por la Ley de Educación Nacional $\mathrm{N}^{\circ}$ 26.206: Primario, secundario, terciario, universitario, centros habilitados para el Plan Fines del Ministerio de Educación, Bachilleratos Populares y Centros de Formación Profesional registrados ante el Ministerio de Educación. Establece cierta flexibilidad, ya que abarca la terminalidad de los distintos tramos educativos no solo primario, secundario sino también superiores, terciario y universitario, e incluye distintas instituciones educativas.

Vinculado a lo laboral, contempla la asistencia para la formación profesional, orientación e intermediación laboral incluyendo aquellos cursos de Orientación e Introducción al Mundo del Trabajo del Ministerio de Trabajo, Empleo y Seguridad Social (MTEySS). También, forma parte de esta oferta la inscripción en el entrenamiento para el trabajo, como prácticas en ambientes de trabajo que incluyen procesos de formación y tutoría. El programa de Inserción Laboral, que promueve la inserción en empleos de calidad, mediante la implementación de incentivos económicos a las empresas ${ }^{[11]}$.

Como otros componentes, el programa ofrece una red de tutores y estrategias de cuidado infantil para aquellos jóvenes (padres y madres) a cargo del cuidado de niños. Esto hace al reconocimiento de un sector clave entre jóvenes en situación de mayor vulnerabilidad social (fundamentalmente las jóvenes madres de 18 a 24 años).

Ahora bien, como un recontó, que, sin ser excluyente, da cuenta de diversas perspectivas desde donde se ha abordado el programa puede decirse que existe una serie de análisis y documentos de coyuntura que repasan y hacen una descripción sobre las reglas operativas, normativas y estructura general de esta medida que evidencian las potencialidades de la propuesta (Repetto \& Langou, 2014). Hay análisis que resaltan el alcance de la medida en términos de promoción de justicia distributiva y como expresión del pasaje a un nuevo paradigma ampliado de protección social que implica que la niñez, la adolescencia y la juventud, dejan de considerarse un riesgo para la sociedad (Mazzola, 2014).

Algunos estudios enfatizan en términos del modelo de transferencias condicionadas que representa el Progresar, proponiendo aproximaciones al diagnóstico de la situación del sector en la etapa de su lanzamiento y (o) estimando la ampliación de oportunidades. (Aguirre \& Marzonetto, 2014; Di Giovambattista, Gallo, \& Panigo, 2014), mientras que otros lo analizan poniendo especial atención a la convergencia de la propuesta con otros programas destinados a la población joven aplicados en países latinoamericanos como PROSPERA de México, PROJOVEN integrado de Brasil, AVANCEMOS en Costa Rica. (D’Alessandre \& Duer, 2015). Mas allá de esta breve reseña, no hallamos materiales oficiales que hallan profundizado y sistematizado información sobre este programa y su evolución.

Resta apuntar que además a pocos años de su puesta en marcha, el 30 de enero del 2018, el presidente Argentino Mauricio Macri, conferencia de prensa mediante, presentó una serie de modificaciones que aun sin revertir la vigencia del Progresar alteran su fisonomía. En principio se anuncia como un programa de Becas Progresar que deja de formar parte de la órbita del ANSES para pasar a ser coordinado desde el Ministerio de Educación. Entre otras reorientaciones, se ha cambiado la franja etaria a quienes está destinado, ya que contemplara de 18 a 30 años, es decir, en el caso de los estudiantes de educación superior no universitaria y universitarios la edad puede extenderse hasta los 30 años. Además, hay nuevas reorientaciones donde el foco está puesto en reforzar el seguimiento del rendimiento escolar. El buen desempeño escolar es evaluado y se vuelve doblemente en una clave tanto para dar continuidad a la beca como para obtener un plus monetario, por ejemplo, privilegiando al alumnado de mayores promedios, entre los universitarios ${ }^{[13]}$ (Decreto (PE) No 90/2018). 
Las becas Progresar se instituyen en la misma línea de reforzar el esquema de oportunidades para la finalización de los estudios en distintos niveles y tramos educativos sin embargo en la actualidad claramente se promueven y acentúan los rasgos meritocráticos para el acceso y continuidad en la asignación de estos recursos estatales y, por ejemplo, la promoción al esfuerzo legitima un esquema escalonado de asignaciones. No obstante, dado el periodo temporal del desarrollo del trabajo de campo de esta investigación, así como del objetivo de este texto no nos detendremos en el análisis de las implicancias de la reconfiguración del programa.

\section{Opiniones y Debates: El Progresar una política joven}

Hasta aquí bosquejamos el marco de cambios en las orientaciones y lineamientos de políticas públicas en el cual se inscribe la creación e implementación del programa Progresar y dimos cuenta de los principales ejes de su arquitectura. En lo que sigue enfocaremos en construir un análisis sobre las opiniones de los actores (gestores y expertos académicos) consultados a lo largo de la investigación.

Nos centraremos en algunos aspectos que entendemos destacables, no solo en lo que hace a esta política específica sino entendiéndola como parte de la trama de medidas dirigidas a esta franja etarea. Siguiendo el esquema general de la propuesta, en el marco de las políticas destinadas a la juventud, se buscó trazar posiciones respecto al esquema de protecciones sociales, el programa y su implementación, así como las tensiones que los atraviesan.

En un primer nivel de análisis se identificaron puntos de confluencia en términos de la propuesta del Progresar su importancia y valoración en materia de políticas de juventud implementadas hasta el momento. En este sentido, un punto de convergencia que vertebra las opiniones es la visión positiva del alcance y cobertura del programa, lo cual resulto una notoriedad para todo el espectro de los actores consultados ${ }^{[14]}$.

Todos los entrevistados hicieron referencia a las estimaciones sobre la cobertura del programa, destacando su dimensión y alcance. Para algunos la masividad del programa es señal indicativa del esfuerzo del Estado Nacional por atender a una problemática en ascenso y poco visibilizada hasta el momento, vale decir, una deuda pendiente en materia de intervenciones de políticas públicas. El tema de las juventudes y su presencia en la agenda política, así como la emergencia de esta intervención destinada a la franja de 18 a 24 años, fue considerada por su significativa amplitud que contrasta y (o) marca una diferencia con las antecesoras experiencias.

Esto de la mano del presupuesto y la decisión política que lo avaló, ya que en sus orígenes las estimaciones oficiales del programa proponían que llegaría a abarcar un total de 1,5 millones de jóvenes.

Una que viene a fortalecer algo que no estaba con mayor presupuesto, tratar de atender lo que tiene que ver con la finalización de estudios básicos, y formación de los jóvenes. Los programas que históricamente teníamos en Argentina tenían pequeños alcances y pequeñas partidas presupuestarias. Entonces si vos decías en invertir y te planteaban el horizonte llegar a 1.500.000 de chicos, estás planteando una política con dimensiones muy diferentes a las que históricamente se fueron planteando a estas poblaciones. (Ev. Nº 1 , Rosana, Mujer, Experta académica).

En correspondencia con lo destacado por buena parte de los entrevistados, en efecto, por la expansión de su cobertura, el Progresar, fue y sigue siendo aún el principal programa de transferencias condicionadas destinado a la población de 18 a 24 años. Asimismo, algunos expertos hilvanan lo positivo del incremento respecto del alcance de la cobertura de esta medida ponderando positivamente sus efectos en términos de justicia distributiva es decir entendiendo la pertinencia del modelo de transferencia condicionada en base a un intercambio monetario directo.

Entonces vos partís del presupuesto, apoyando dos cuestiones. Una que tiene que ver con promover igualdades de oportunidades, pero también hacer justicia distributiva en términos económicos porque los chicos no van a poder salir por sí mismos de esta situación por más que uno lo forme, o sea, cierto apoyo monetario se necesita. (Ev. $\mathrm{N}^{\circ} 1$, Rosana, Mujer, Experta académica). 
En breves palabras puede decirse que uno de los aspectos destacados por los expertos estuvo en relación directa con la amplitud de esta política en función del volumen de población joven que potencialmente estimaba abarcar. Lo cual remite a cierto avance en términos de visibilidad de la franja etaria, así como también a las acuciantes y complejas problemáticas que enfrenta la juventud del país, al mismo tiempo y como contracara muestra cierta ausencia hasta el momento de políticas más integrales para el sector en la agenda de las políticas públicas nacionales.

El alcance de la cobertura del programa se conjuga con otra notoriedad que ha sido señalada por los entrevistados como lo es la incorporación desde el decreto de creación del programa sobre la concepción del joven como un sujeto de derecho. Es decir, la expresa adopción del enfoque de derechos y una mirada integral de las múltiples problemáticas que los atraviesan, tomando en cuenta además la diversificación de las trayectorias socioeducativas y laborales del sector.

En este sentido, la materialización de la propuesta del Progresar parece haber sido relevante en tanto desde su diseño deja enunciada no solo la perspectiva de derecho como eje orientador de la intervención, sino también expresa lo necesario de una mirada integral ante la complejidad de las problemáticas que enfrentan los jóvenes en situación de vulnerabilidad económica.

Una política innovadora en materia de juventud, porque se los trata como un sujeto de derecho que estaba pendiente porque no se los visibilizó. Y el Progresar justamente lo que viene es a visibilizar a un sector etario que estuvo postergado dentro de las políticas públicas y las políticas sociales. Haber logrado implementar una política que esté destinada a este segmento me parece que primero es reconocerlos como sujetos de derechos. (Ev. N6, Ana, Mujer, Gestora).

Se instaló que hay un programa que es para jóvenes y que se llama Progresar. Es un derecho ganado y se va a pelear. Es como un hito. Entonces yo creo que es algo que se armó para que los pibes peleen esa bandera de acá a largo plazo. (Ev. Nº10, Lucía, Mujer, Gestora)

Una política innovadora en materia de juventud, porque se los trata como un sujeto de derecho que estaba pendiente porque
no se los visibilizó. Y el Progresar justamente lo que viene es a visibilizar a un sector etario que estuvo postergado dentro de
las políticas públicas y las políticas sociales. Haber logrado implementar una política que esté destinada a este segmento me
parece que primero es reconocerlos como sujetos de derechos. (Ev. Nº, Ana, Mujer, Gestora).
Se instaló que hay un programa que es para jóvenes y que se llama Progresar. Es un derecho ganado y se va a pelear. Es
como un hito. Entonces yo creo que es algo que se armó para que los pibes peleen esa bandera de acá a largo plazo. (Ev. N
${ }^{\circ} 10$, Lucía, Mujer, Gestora)

El carácter del enfoque de derecho reenvía a los compromisos que el Estado asume como parte de las acciones estatales. La incorporación desde el marco técnico-operativo de este enfoque de derechos es señalada como un aspecto positivo por el conjunto de los entrevistados. En un mismo sentido, algunos expertos valuaron el lanzamiento del Progresar entendiendo que los lineamientos del programa apuntaron a avanzar básicamente hacia la amplitud de oportunidades y ello logro instalarse en la escena de las demandas de la propia población de jóvenes del país.

Finalmente, otro elemento recurrente en las respuestas ha sido la señalización sobre la inclusión de componentes "novedosos" del programa. En esta dirección destaca el rol de los tutores como un aspecto significativo en el acompañamiento de las trayectorias de los jóvenes, y, sobre todo la inclusión de espacios de cuidado de niños (niñas) - guarderías -, como una posibilidad ausente en programas anteriores.

Así, del análisis resulta la convergencia de las opiniones en términos de destacar dos componentes: Las guarderías y las tutorías como elementos positivos de la propuesta. Destaca, por un lado, el acompañamiento y seguimiento para el destinatario de la política a través de tutorías incentivando la participación, y por otro, la posibilidad de gestionar espacios de cuidado de niños (niñas) para aquellos jóvenes que tienen hijos a cargo, de modo que el programa más allá de la transferencia monetaria contempla una trama de aspectos que lo hacen destacable. 
[...] me parece muy interesante y hasta donde entiendo no lo han logrado resolver es la de hacer un acompañamiento con jardines maternales para los hijos de las mujeres que dejaron en buena medida porque tuvieron familia. Es una de las causas de mayor incidencia en la deserción escolar en las mujeres y en los varones en alguna medida también. Esa idea de articular un programa de finalización de la escolarización con acompañamiento a los chicos a través de jardines maternales me parece muy innovador y de funcionar bien tendría muchas potencialidades. (Ev. No 2, Julio, Varón, Experto académico).

Más allá de una transferencia monetaria, tanto el acompañamiento y seguimiento (a través de tutorías, incentivando la participación) por parte del Estado sobre qué pasa con el destinatario de la política una vez que la recibe, y la apertura de nuevos componentes como la inclusión de guarderías que expresan la comprensión de un aspecto incidente en la problemática del abandono escolar entre los y sobre todo las jóvenes, suman aspectos valuados positivamente por los expertos consultados. Es más, las guarderías, en nuestro país resultan un componente sui generis en un programa de alcance masivo como el Progresar, aunque como contracara de la alta valuación no se han constatado avances respecto a su instrumentación en lo que va de los años de implementación del programa.

\section{Lo SIMPle Y LO COMPLeJo: Políticas PÚBliCAS Y PENDIENTES}

En una segunda dimensión de análisis nos propusimos poner el eje en cuestiones pendientes en la agenda joven. Ampliando el espectro de los aspectos circunscriptos al programa que ha sido el foco de nuestro análisis, en este último apartado vinculamos una suerte de opiniones de los actores consultados y sus apreciaciones acerca de una serie de aspectos que pueden ser tanto debilidades y (o) cuestiones no contempladas en el marco del Progresar como así también otros que lo exceden y transcienden, dejamos asíla visión sobre la singularidad de una medida para plantear una reflexión sobre tensiones y desafíos para repensar transformaciones en materia de políticas de juventud.

En perspectiva regional las opiniones recogidas indican que la agenda de las políticas de juventudes actuales reclama un horizonte de mayor atención de consignas y acciones que no confluyen con la implementación de intervenciones aisladas. A través de sus opiniones los consultados dan cuenta de una mirada acerca de las cuestiones relativas a las tensiones latentes entre políticas integrales y desafíos pendientes de aspectos que consideran una suerte de limitaciones que están presentes en materia de intervenciones dirigidas a los jóvenes.

Considerando el horizonte de enfoque de derechos como paradigma que, como hemos referido, ha cobrado cierto impulso en el contexto regional y nacional de la última década en particular en relación con la población de niñas (nños) y jóvenes, una tensión refiere a la ampliación de los sistemas de seguridad social. En este sentido, las reflexiones convocan a cuestionar el carácter instituyente de políticas destinadas al sector joven entendiendo, la importancia de un nuevo piso institucional de seguridad social que permita dotar de sustancia y sustentabilidad al enfoque de derechos.

En Argentina y en todos los países latinoamericanos, nosotros tenemos pendiente una agenda de cómo ampliamos la construcción de seguridad social, vinculada no sólo a las prestaciones consecutivas para que un programa de este tipo podría ser no un programa más vinculada a la transferencia condicionada sino vinculado a un derecho de seguridad social para todos los jóvenes. Con participación, cruzada con lo cultural, el deporte, virando a otro enfoque no tan tradicionalista del programa. (Ev. $\mathrm{N}^{\circ} 1$, Rosana, Mujer, Experta académica).

Otro punto que destaca en este testimonio, también mencionado por otros de nuestros entrevistados, es el que se refiere a la incorporación de actividades en relación con lo cultural y lo deportivo como parte de distintos espacios de acción social que podrían conjugarse en las actividades programáticas y los dispositivos de intervención de una misma medida o programa. Esta ampliación comulgaría tanto con la intención de superar una mirada fragmentada, como con la posibilidad de trascender las ofertas tradicionales vinculadas a las líneas de educación-trabajo. Estas últimas sin perder la condición de prioritarias podrían conjugarse con distintas actividades compatibles incluso con prácticas informales frecuentes en las culturas más propias a los jóvenes contemporáneos. Es decir, apunta a la posibilidad de construcciones que sin anularlas exceden 
el esquema tradicional de las líneas de educación y trabajo, comprendiendo oportunidades de desarrollo en otras áreas de acción y formación ya sean consideradas en forma exclusiva y (o) complementaria.

Otro aspecto interesante es acerca de la participación como un pendiente ineludible. Pensar una política de Estado destinada al sector poblacional de los jóvenes contemporáneos promoviendo oportunidades de inclusión social comulga con la idea - a esta altura poco novedosa pero escasamente activada - en el respeto a la participación de los mismos sujetos destinatarios de las acciones programáticas.

Hay mucha estigmatización de los jóvenes como vagos, como faloperos, violentos. O se ponen problemas de la sociedad como si fuesen problemas de la juventud en todo caso. Yo creo en la juventud. Y creo todo lo contrario que los jóvenes no son violentos, son menos sectarios, menos discriminadores que otras generaciones por procesos históricos. Somos una democracia joven, pero creo que los que nacimos en democracia tenemos una formación y una idiosincrasia en la que yo creo y apuesto mucho. Me parece que cualquier política de Estado tiene que pensarse en tomarse en conjunto con el sector que se está involucrando. (Ev. No11, Nicolás, Varón, Gestor).

Yo creo que toda política tiene que tender a generar a ampliar los marcos de democracia. Entendiendo a la democracia como ampliar la participación, más inclusión. Los jóvenes son una población vulnerable en términos de trabajo, en términos de educación, sociales, económicos. Me parece que lo que tendría que pensar una política de Estado sea cual sea desde el sector que se esté pensando, lo que tiene que hacer es ampliar los márgenes de participación, de inclusión de los jóvenes. (Ev. No11, Nicolás, Varón, Gestor).

Finalmente, a la hora de emprender el diseño y la construcción de la política, con una mirada transversal e integral, otro punto de interés reside en la recuperación de otras redes sociales y tejidos de proximidad. Ello pone de relieve un aspecto que consideramos sustantivo, dado que trasluce la importancia de no pensar en una política aislada sino por el contrario, la construcción de redes y tramas que la conforman. En este sentido, rescatamos la importancia de repensar intervenciones que en un horizonte amplio tiendan a la reconstitución de tejidos sociales de proximidad que permitan una dinámica de intercambios óptima, un círculo virtuoso para la generación de oportunidades de enlace entre ámbitos y actores sociales promoviendo vinculaciones intra e intergeneracional auspiciosas.

\begin{abstract}
Me parece que nosotros lo que tenemos que recuperar son las organizaciones civiles, las organizaciones no gubernamentales, que permitan que el Estado pueda tener un diálogo con el resto de la sociedad de una manera más organizada. Creo que ahí los clubes, los centros de estudiantes, los sindicatos, me parece que ahí hay que empezar a democratizar ciertos ámbitos, empezar a participar en ciertos ámbitos que permiten que la sociedad se vaya organizando. Me parece que se recuperó el Estado, pero en algún punto quedó recuperar algunos ámbitos de la sociedad civil. Que eso permite tener un diálogo más organizado, más constructivo y que además te permite cuando perdés el Estado no pierdas todo. Yo creo que igual seguimos en esa construcción, me parece que hay que seguir trabajando. Yo creo que sí se puede organizar más allá de lo que después se haga concretamente me parece que lo primero es recuperar ciertos ámbitos, generar otros ámbitos también. (Ev. No11, Nicolás, Varón, Gestor)
\end{abstract}

\title{
Consideraciones Finales
}

En el marco de una región atravesada por desigualdades, crisis y emergencias sociales recurrentes, la revisión de intervenciones sociales destinadas a los jóvenes son materia de vital interés tanto considerando la situación actual como el horizonte de las nuevas generaciones. Enfocando en el panorama regional, expertos como Cechini (2015) indican que en la actualidad se han expandido las acciones de los PTC dirigidas a la juventud que se concentran fundamentalmente en las áreas de educación y trabajo, no obstante, las iniciativas encuentran sus límites de corto alcance en tanto el mercado laboral no ofrezca oportunidades de inserción a los jóvenes o no se logre avanzar en una educación de calidad (Cechini, 2015).

Este texto tuvo como intención aportar al debate a partir de un análisis enfocado en una medida relativamente reciente en el contexto, el Progresar. En Argentina, en particular en la última década del presente siglo, los programas destinados a la población de jóvenes se han reforzado fundamentalmente con el referido programa. Este programa desde sus inicios tiene como horizonte la terminalidad educativa y la 
capacitación y orientación en la búsqueda de empleo, es decir que combina dos lógicas de acción en una misma política. Al mismo tiempo, para Argentina, suma la más ambiciosa política de promoción representando un desafío en cuanto a la extensión de la cobertura.

Como hemos advertido, existen ciertos rasgos innovadores en el lanzamiento del referido programa, rasgos de su arquitectura que permiten distinguir un avance en el reconocimiento de la necesidad de considerar la multidimensionalidad de problemáticas que afectan a los jóvenes y el desafío que ello conlleva. Además, al menos desde el diseño técnico-normativo, entiende al joven como sujeto de derecho y conjuga una serie de componentes de acompañamiento como tutorías y guarderías para jóvenes madres y padres, facilitando así la inclusión de estos en las iniciativas desplegadas.

A la luz del análisis, cuestiones como la masividad y el reconocimiento del joven como sujeto de derechos fueron aspectos destacados y valuados positivamente por los entrevistados. Destaquemos entonces que buena parte de los testimonios expuestos como parte del trabajo de investigación realizado abonan a la idea de la relevancia de una política destinada al sector poblacional joven. En esta dirección, en base a este desarrollo, puede decirse que el Progresar se trata de una política dirigida a un sector históricamente postergado en materia de intervenciones de este tipo. Además, resultó una política que en sus inicios planteó un alcance de gran dimensión respecto a aquellas que se implementaron con anterioridad en el esquema argentino para dicho sector. Retomando palabras de una de nuestras entrevistadas, "Un sector etario que estuvo postergado dentro de las políticas públicas y las políticas sociales.”.

Suma a ello, otra cuestión substantiva que es que el Progresar se instaura como una política que desde su mismo decreto de creación enuncia la perspectiva de derecho como eje orientador de sus acciones programáticas. Incluso, siguiendo lo dicho por una gestora entrevistada, "Una política que esté destinada a este segmento me parece que primero es reconocerlos como sujetos de derechos".

Ahora bien, posicionar a los jóvenes como sujetos de derecho conlleva en sí mismo tanto un reconocimiento como una responsabilidad ineludible del Estado en tanto garante de los mismos, lo cual retoma y expresa lo necesario de una mirada integral ante la complejidad de las problemáticas que enfrentan los jóvenes en situación de vulnerabilidad económica.

Los desafíos en torno a las políticas públicas destinadas a la población joven constelan un conjunto de múltiples cuestiones. Retomar la noción de integralidad como horizonte alcanzable, no solo conceptualmente sino como parte del paradigma de derechos bajo el cual tiene surgimiento las acciones emprendidas por el estado para dar respuesta a problemáticas juveniles es sustantivo y vertebral, pero aun dista de traducirse en una realidad palpable. Ciertamente el programa analizado en buena medida depende de garantizar el funcionamiento de la salud y educación con solidos sistemas universales, pero al mismo tiempo de la continuidad y la trama institucional que logre generar y profundizar los avances en materia de amplitud de protección social integral, hacia un sector claramente postergado.

En este marco son de vital importancia las acciones estatales orientadas a la promoción de los derechos de los jóvenes, considerando estrategias múltiples para enfrentar la complejidad y variada gama de problemáticas que en distintas combinaciones enfrentan las actuales generaciones de jóvenes como causas que condicionan sus trayectorias.

\section{BiBLiografía}

Aguirre, J., \& Marzonetto, G. (2014). Sin estudio y sin trabajo. El Programa de Respaldo a Estudiantes Argentinos: PROG.R.ES.AR. Serie Análisis de Coyuntura, Doc. № 25, Centro Interdisciplinario para el estudio de políticas públicas (CIEPP), Buenos Aires, Argentina.

Becher, Y. (2016). Políticas de juventud(es): entre la autonomía y la asistencia. Un análisis desde el enfoque de derechos. Revista Administración Pública y Sociedad, 1(2). 38-59. 
Bertranou, F., \& Casanova, L. (2015). Trayectoria hacia el trabajo decente de los jóvenes en Argentina: contribuciones de las politicas públicas de educación, formación para el trabajo y protección social. OIT, Ginebra. Recuperado de: https://www.ilo.org/wcmsp5/groups/public/---americas/---ro-lima/---ilo-buenos_aires/documents/public ation/wcms_454395.pdf

Cechini, S. (2015). Programas de Transferencias Condicionadas: panorama regional y su impacto en la población. SITEAL/IIPE/Unesco, Sede Regional Buenos Aires, Argentina.

Cecchini, S. (2014), "Social protection, poverty and inequality: a comparative perspective", Journal of Southeast Asian Economies, 31 (1). pp. 18-39.

Cecchini, S. y R., Martínez (2011). Protección social inclusiva en América Latina: Una mirada integral, un enfoque de derechos, Libro de la CEPAL No 111, CEPAL, Santiago de Chile, Chile. Recuperado de: https://repositorio.ce pal.org/bitstream/handle/11362/2593/S2011914_es.pdf?sequence=1\&isAllowed=y.

Cepal/OIJ (2014). Invertir para transformar: la juventud como protagonista del desarrollo CEPAL/OIJ. Santiago de Chile, Chile. Recuperado de: https://repositorio.cepal.org/bitstream/handle/11362/39314/1/invertirparatra nsformar.pdf.

D'Alessandre, V., \& Duer, C. (2015). Programas de Transferencias Condicionadas orientados a jóvenes. El caso del PROGRESAR. Cuaderno $n^{\circ}$ 22, IIPE - Unesco Sede Regional Buenos Aires / Organización de Estados Iberoamericanos Para la Educación, la Ciencia y la Cultura (OEI).

Danani, C. (2016). Las políticas públicas del área de desarrollo social durante los gobiernos de Néstor Kirchner y Cristina Fernández. Análisis n ${ }^{\circ} 12$. Fundación Friedrich Ebert. Buenos Aires, Argentina.

Di Giovambattista, A. P., Gallo, P., \& Panigo, D. (2014). Empleo, desempleo \& politicas de empleo El impacto distributivo del "PROG.R.ES.AR" en Argentina. Una primera aproximación en base a microsimulaciones. $\mathrm{N}^{\circ}$ 17. Primer semestre 2014. Centro de Estudios e Investigaciones Laborales (CEIL) - Consejo Nacional de Investigaciones Cientificas y Técnicas (CONICET), Buenos Aires, Argentina. Recuperado de: https://ri.conicet .gov.ar/handle/11336/4543.

Friedrich, M. C. R., Machado Benite; A. M., \& Canavarro Benite (2012). O Programa Nacional de Inclusão de Jovens: Projovem: uma análise entre a proposta oficial e a experiência vivida em Goiânia, Ensaio: Aval. Pol. Públ. Educ., 20(74), 185-206.

Jacinto, C. (2016). De los derechos a las garantías en las transiciones de los jóvenes al empleo Alcances y límites de las tramas entre educación secundaria, formación para el trabajo y protección social. En: Jacinto, C. (coord.) (2016). Protección social y formación para el trabajo de jóvenes en la Argentina reciente: entramados, alcances y tensiones, Libros Instituto de Desarrollo Económico y Social (IDES), Buenos Aires, Argentina. 9-30.

Kaplan, D., \& Levy, S. (2014). The evolution of social security systems in Latin America, Social Insurance, Informality, and Labor Markets: How to Protect Workers while Creating Good Jobs, M. Frolich y otros (eds.), Nueva York, Oxford University Press.

Marzonetto, G., \& Aguirre, J. (2014). Sin estudio y sin trabajo. El Programa de Respaldo a Estudiantes Argentinos: PROG.R.ES.AR. Serie Análisis de Coyuntura $n^{\circ}$ 25, Centro Interdisciplinario para el Estudio de Políticas Públicas (CIEPP). Buenos Aires, Argentina.

Mazzola, R (2014). PROGRESAR: Juventudes, bienes públicos y justicia distributiva. Revista Estado y Politicas Públicas, 2(2), 91-113.

Ocampo, J. A., \& Gómez-Arteaga, N. (2017). Los sistemas de protección social, la redistribución y el crecimiento en América Latina. Revista CEPAL, (122)1,7-34.

Otero, A. (2017). Agenda joven en Argentina. El caso del Programa de Respaldo a Estudiantes de Argentina PROG.RE.S.AR. Metamorfosis, (6)1, 62-75.

Repetto, F., \& Díaz Langou, G. (2014). Recomendaciones integrales de política pública para las juventudes en la Argentina. Doc. de Politicas Públicas n ${ }^{\circ} 137$, Centro de Implementación de Políticas Públicas para la Equidad y el Crecimiento (CIPPEC), Buenos Aires, Argentina.

Rodríguez U.J., \& Giambroni, L. (2015). Avances y desafíos de las Políticas de Juventud en el siglo XXI. Revista Estado y Politicas Públicas, 3(4), 152-165. 


\section{Notas}

[1]Rastreando los antecedentes programáticos en el seno del Ministerio de Trabajo, Empleo y Seguridad Social (MTEySS), un primer programa dirigido a atender educación-trabajo originado desde este ministerio fue el "Proyecto Joven", posteriormente se aplicó el programa "Capacitar", ambos contaban con el apoyo financiero del BID. El MTEySS amplió sus acciones en la década de 1990 asumiendo gradualmente nuevas funciones, como la elaboración de programas de formación profesional, capacitación, etc., que antes eran implementados desde el Ministerio de Educación. (Otero, 2017, p. 68).

[2]Durante el año 2005 se sanciona la Ley 26.061 de Protección Integral de los Derechos de Niños y Niñas y Adolescentes que abole el sistema de tutela Estatal basado en el Patronato.

[3]A mediados del año 2015, más de 3,5 millones de niños y adolescentes se encontraban cubiertos por la AUH.

[4]A partir de mayo de 2011, las prestaciones AUH se complementaron con el lanzamiento de la "Asignación Universal por Embarazo (AUE) para protección social”, que se otorga a las futuras madres desde las doce o más semanas de gestación.

[5]El Programa Jóvenes con Más y Mejor Trabajo (PJMYMT) (2008) está destinado a jóvenes de 18 a 24 años que no terminaron sus estudios primarios o secundarios y a promover oportunidades de inclusión social y laboral por medio de diferentes acciones que posibiliten finalizar estudios obligatorios y construir un perfil profesional, realizar experiencias de formación, practicas calificantes, iniciar una actividad productiva independiente y $(\mathrm{o})$ insertarse en un empleo.

[6]A diferencia de la AUH ningún componente del Progresar forma parte del Sistema de Seguridad Social (Danani, 2016). Además, tanto la administración como el pago se viabiliza a través de la agencia ANSES a lo largo de todo el territorio nacional pero su financiamiento depende del Tesoro Nacional.

[7]El Plan de Finalización de Estudios Primarios y Secundarios (FinES) es un plan argentino para finalización de la Escuela Primaria y Escuela Secundaria llevado a cabo por el gobierno nacional a partir del año 2008, de alcance nacional que funcionó en todas las jurisdicciones de Argentina. En el año 2010, se creó el Plan de Finalización de Estudios Primarios y Secundarios 2 (FinES).

[8]La condicionalidad refiere a que los jóvenes deben presentar las acreditaciones correspondientes, certificados de asistencia, acreditaciones de los espacios institucionales en los cuales se incluyan, etc.

[9]Refiere a la articulación con el Programa Sumar (2012), con origen en el Ministerio de Salud de la Nación.

[10]El monto a pagar a los beneficiarios consiste en una suma no contributiva y mensual según establezca oportunamente el Ministerio de Educación y siguiendo el Art 20 del Decreto (PE) No 84/2014 (BO 27/01/2014). "El Programa se financiará con las partidas que anualmente asigne la Ley de Presupuesto con fondos provenientes del Tesoro Nacional”.

[11]Mediante ello promueve espacios de articulación con el Programa Jóvenes con Más y Mejor Trabajo (MTEySS). También, se estableció que los jóvenes inscriptos en el Progresar también pueden formar parte del Programa Empalme y Programa Construir Empleo ambos con origen en el MTEySS.

[12]Es interesante destacar que este programa guarda ciertos puntos de conexión con la estructura del programa Progresar aquí analizado. Un análisis sobre la implementación del Projoven en Goiânia puede verse en Friedrich; Machado Benite y Canavarro Benite (2012).

[13]Algunas especificaciones, sigue destinado a jóvenes de 24 años hasta 30 años que busquen completar estudios primarios y (o) secundarios y para acceder cada año deben pasar al año escolar siguiente por lo cual, en caso de repetir, pierden el derecho. El monto de la beca varía según el nivel educativo y en el caso universitario o terciario, si la carrera es estratégica, el año que se curse; el promedio de calificaciones; la aprobación de materias obtenidas. Para universitario o terciario, para acceder a la beca por primera vez hay que contar con el 50\% de las materias aprobadas que le correspondan hasta el momento mientras que, para dar continuidad al beneficio cada año deben aprobar el $50 \%$ más una materia del total teórico de materias del año cursado. Además, los estudiantes universitarios becados que aprueben el total de las materias de cada año con un promedio mayor a 8 recibirán en marzo una distinción y un bono equivalente al monto total de la beca del año transcurrido. El buen desempeño escolar es evaluado y se vuelve doblemente en una clave tanto para dar continuidad a la beca como para obtener un plus monetario privilegiando al alumnado de mayores promedios (entre los universitarios). Esto se suma a las estrategias que refuerzan la elección de carreras concebidas como "estratégicas" para el desarrollo del país que reciben un monto en escalafones mayor. La imposición de mayores criterios meritocráticos para la participación y continuidad promueve un acceso segmentado a la protección social. 
[14]Si bien el PJMyMT, antecesor más directo y próximo logró constituirse en una intervención relevante y de magnitud en términos de la población destinataria de las políticas dirigidas a la franja etaria de 18 a 24 años del país, el Progresar constela un esfuerzo mayor. Como han señalado "en sus inicios las estimaciones oficiales del programa proponían que llegaría a abarcar un total de 1,5 millones de jóvenes, lo cual significaba un 30\% de los 4 millones de jóvenes de 18 a 24 años del país. Para enero del 2015 había incorporado un aproximado de 565 mil jóvenes. Luego en junio del presente año considerando el total país eran 702.867 aquellos que accedían a la prestación por haber acreditado la escolaridad. En cuanto a la composición del total de titulares que reciben la prestación, el 63\% son mujeres y el 78\% tiene 22 años o menos. Mientras que la distribución territorial indicaba que, Buenos Aires era la provincia con mayor cantidad de casos (232.872), seguida por Salta (48.809) y Tucumán (39.171). En cuanto a las actividades, es decir, qué hacen esos jóvenes, la mayor parte está cursando estudios universitarios (44\%), un 29\% finalizando el secundario, mientras que el 23\% restante se entra inscripto en capacitaciones, cursos y oficios. (Otero, 2017).

\section{BY-NC-ND}

